\title{
Editorial: Key Players in Systemic Sclerosis: The Immune System and Beyond
}

\author{
Philippe Guilpain ${ }^{1,2,3^{*}}$, Danièle Noël ${ }^{2,3}$ and Jérôme Avouac ${ }^{4,5,6}$ \\ ${ }^{1}$ Department of Internal Medicine and Multi-Organic Diseases, Local Referral Center for Systemic and Autoimmune \\ Diseases, St Eloi Hospital, Montpellier, France, ${ }^{2}$ Faculty of Medicine, University of Montpellier, Montpellier, France, ${ }^{3}$ Inserm \\ U1183, Institute for Regenerative Medicine and Biotherapy (IRMB), St Eloi Hospital, Montpellier, France, ${ }^{4}$ Department of \\ Rheumatology, Cochin Hospital, AP-HP.CUP, Paris, France, ${ }^{5}$ Faculty of Medicine, University of Paris, Université Paris \\ Descartes, Paris, France, 6 INSERM U1016 and CNRS UMR8104, Cochin Institute, Paris, France
}

Keywords: systemic sclerosis, pathophysiology, co-stimulation, abatacept, innate immune system, mesenchymal stromal cell, extracellular vesicles, cancer

\section{Editorial on the Research Topic}

Key Players in Systemic Sclerosis: The Immune System and Beyond

\section{OPEN ACCESS}

Edited and reviewed by: Herman Waldmann, University of Oxford, United Kingdom

*Correspondence: Philippe Guilpain p-guilpain@chu-montpellier.fr

Specialty section: This article was submitted to Immunological Tolerance and Regulation,

a section of the journal

Frontiers in Immunology

Received: 03 September 2021 Accepted: 07 September 2021 Published: 30 September 2021

Citation:

Guilpain P, Noël D and Avouac J (2021) Editorial: Key Players in Systemic Sclerosis: The Immune

System and Beyond.

Front. Immunol. 12:770419. doi: 10.3389/fimmu.2021.770419
In these times of Covid-19 pandemic, during which medical research is mobilized in the fight against the coronavirus, patients continue to suffer from other acute and chronic diseases and research on them must continue. Systemic sclerosis (SSc) is one of these diseases, both chronic and complex, whose evolution can be fatal and whose clinical repercussions, variable from one patient to another, can result in a profound alteration of autonomy and quality of life. The current crisis of the coronavirus should not make us forget this reality.

At this point, we must recall the well-known generalities about this disease (1). Systemic Sclerosis is a rare multifaceted disease, whose development is related to the interplay of three major pathophysiological mechanisms, i.e. vascular impairment, autoimmunity and uncontrolled fibrogenesis. These mechanisms lead to the development of the main disease-related manifestations including skin, lung, heart, and gastrointestinal tract involvement. Fibrosis primarily affects the skin dermis. However, the lungs, heart, and/or digestive tract may also be involved. Vascular impairment leads to the development of Raynaud's phenomenon, telangiectasia, digital ulcers, pulmonary arterial hypertension and/or vascular renal crisis. These features render SSc a very peculiar autoimmune disorder. Autoimmunity in SSc is mediated via the immune cell activation and production of autoantibodies (Abs). The latter are mainly directed against three target autoantigens, namely Topoisomerase 1 (anti-SCL70 Abs), CENP-A/B (anti-centromere Abs) or RNA-polymerase III. Sustained immune and inflammatory stimulation promote pathological fibroblast activation, which results in excessive extracellular matrix protein production and accumulation in tissues.

The clinical presentation of the disease is heterogenous. SSc may present in three classical forms, including limited cutaneous, diffuse cutaneous, and 'sine scleroderma' subsets, and may associate with other connective tissue diseases. SSc has a poor prognosis, globally with a severe vital prognosis and potential long-term disability. Its treatment is mainly palliative and based on symptomatic therapies, vasodilators, immunosuppressants, targeted therapies and antifibrotic drugs. Therefore, 
advances in our understanding of the crucial actors and mechanisms that underlie this disease are essential for improving therapy (hopefully, through the development of innovative therapeutic approaches) and patient outcomes.

In fact, a myriad of questions regarding SSc pathogenesis still remain unresolved. By exploring the most recent advances and discoveries in this field, this Research Topic aimed to transition our understanding of SSc pathogenesis from its old conception (based on the three main mechanisms, i.e. autoimmunity, fibrogenesis and vascular involvement) to a modern view.

When we proposed this topic to Frontiers in Immunology (https://www.frontiersin.org/research-topics/7231/key-players-insystemic-sclerosis-the-immune-system-and-beyond\#overview), the most recent advances in SSc had included the following key points (2): (i) fibroblast activation and endothelial impairment are likely dependent on environmental, genetic and epigenetic factors, with a specific contribution of endogenous and/or exogenous oxidative stress; (ii) a link was recently established between cancer and some specific forms of SSc [with particular autoantibodies (i.e. anti-RNA polymerase III and related Abs)], illustrating that the immune response could be directed against malignant targets; (iii) metabolic pathways (such as those implicating PPAR- $\gamma$ ) were also likely important for SSc development and could represent innovative therapeutic targets; (iv) while autologous hematopoietic stem cell transplantation following intensive immunosuppressant therapy had convinced more and more SSc specialists of its interest, progenitor cell-based therapies [such those using mesenchymal stromal cells (MSC)] were under development with great promises; (v) additionally, several pharmacological approaches were also under development to counteract fibrosis and vascular impairment in SSc.

The present Research Topic is entitled "Key players in Systemic Sclerosis - The immune system and beyond...", since key players in SSc are not limited to the immune system. This is one of the most original specificities of the disease. So, in the present Research Topic, we proposed 20 articles (including 10 review articles and 10 original articles) in the field of SSc, focusing on various cellular, molecular and environmental actors involved in SSc, in relationship with the immune system, and on innovative therapies for SSc. So, you can read in the present book, two original articles on Abs [anti-Ro (Gkoutzourelas et al.), and antiphospholipid antibodies (Sobanski et al.)], illustrating the variety of $\mathrm{Ab}$ repertoire in SSc, and discussing the capacity of SSc for overlapping. Herein, you can also find articles on the role of immune system and its interaction with other key players (such as fibroblasts and endothelial cells): the role of the innate immune system (Bhattacharyya et al.; Laurent et al.), the costimulatory pathways (Boleto et al.), the Regulatory T Cells (Frantz et al.), the microchimerism (Di Cristofaro et al.), the relationships between inflammatory cytokines and fibroblasts (Dufour et al.), the induction of endothelial microparticles release by natural killer cells (Benyamine et al.). Otherwise, other key players are presented: the central role of myofibroblasts is extensively reviewed (van Caam et al.), while a novel resistance mechanism to apoptosis is described in fibroblasts and vascular smooth muscle cells (Takata et al.); the deleterious effects of oxidative stress is discussed considering endothelial-to-mesenchymal transition (Thuan et al.), whereas the beneficial role of Nrf2-antioxidant response is illustrated in the murine model of $\mathrm{HOCl}$-induced scleroderma (Kavian et al.; Kavian et al.). The intriguing relationships between SSc and cancer are also presented in a review article (Maria et al.), underlining the similarities between fibrosis and cancer development as well as the most recent data on paraneoplastic forms of SSc. The phenomenon of vascular leaking is also presented as a key player in the early phase of SSc (Bruni et al.), and the stages of fibrosis progression are now documented in the murine $\mathrm{HOCl}$-induced model (Maria et al.). Finally, therapeutic approaches based on autologous hematopoietic stem cell transplantation (Del Papa et al.) or MSC (Maria et al.; Peltzer et al.; Maria et al.; Rozier et al.) are also presented in this book. We thank all the authors for their contribution.

Three years and one pandemic later, the content of this book remains fully relevant and continues to shed light on new ways of understanding and treating SSc. We would now like to highlight some of the data and concepts described in this book, and discuss them in light of other recent studies.

We encourage the reading of the review article on the innate immune system (Laurent et al.). While macrophages and type I interferon have been considered for many years as key players in SSc, several other components of the innate immune system (such as pathogen-recognition receptors, platelet-derived dangerassociated molecular patterns, innate lymphoid cells, and plasmacytoid dendritic cells) exhibit now an emerging role. Research on the subject is ongoing and promising. A recent study (3) demonstrates the role of innate lymphoid cells-2 (ILC2) both in human and murine SSc, and more precisely the interplay between these cells and cytokines (namely, TGF $\beta$ and IL10) in the development of fibrosis. Interestingly, TGF $\beta$ could induce the switch of ILC2 from an 'inflammatory' phenotype $\left(K L R G 1^{\text {high }}\right)$ to a 'natural' phenotype (KLRG1 ${ }^{\text {low }}$ ), which exhibits a more potent capacity for fibrogenesis. Even more interesting, pharmacological TGF $\beta$ blockade with the anti-fibrotic agent pirfenidone did not significantly reduce the fibrotic process, while a combined treatment with pirfenidone and IL10 did. This therapeutic response was associated with a reduced number of skin infiltrating ILC2 and an enhanced expression of KLRG1. These findings illustrate the complexity of the disease as well as the variety of interactions between key players, both at the molecular and cellular levels, and suggest that combined therapies may be the future.

T-cell costimulation pathways (such as CD28/CTLA-4, ICOS-B7RP1, CD70-CD27, CD40-CD154, or OX40-OX40L) may be key players in the pathogenesis of SSc, as suggested by several experimental findings (Boleto et al.). Notably, the blockade of T-cell costimulation with abatacept (CTLA-4-Ig) improved the various manifestations of SSc in pre-clinical animal models $(4,5)$ and these promising results were corroborated by some preliminary clinical reports $(6,7)$. Interestingly, abatacept prevented the development of inflammation-driven fibrosis, but demonstrated no efficacy in the treatment of late and noninflammatory dermal fibrosis (4). Unfortunately, a recent trial (ASSET trial, NCT02161406) did not confirm the promises 
ofabatacept for patients with early diffuse SSc, considering the modified Rodnan skin thickness score (mRSS) at 12 months as primary endpoint (8). Nevertheless, according to this trial, abatacept remains promising when considering some secondary endpoints, i.e. the "Health Assessment Questionnaire disability index" (HAQ) and the composite score called "American College of Rheumatology Combined Response Index in diffuse cutaneous Systemic Sclerosis (ACR CRISS)". These two scores reflect respectively the disability induced by SSc and the probability of improvement in response to treatment in patients with early diffuse cutaneous SSc. Undoubtedly, all these findings illustrate also the complexity and heterogeneity of the disease, as well as the difficulty to monitor SSc and to determine the different components of the disease for a given patient (inflammatory versus dry fibrosis, for example). It also reminds us that encouraging results obtained in animals do not presume a success in humans. So, these -almost- negative results with abatacept suggest that CD28 pathway inhibition alone is insufficient to significantly impact skin disease in patients with early diffuse SSc, and suggest the co-targeting of CD28 with another costimulatory molecule to gain efficacy. For instance, the development of a dual CD28/ICOS antagonist (ALPN-101) may be of interest, since the blockade of both CD28 and ICOS in an acute graft versus host mouse model led to improved survival in ALPN-101-treated mice compared to mice receiving a CD28CD80/CD86 pathway antagonist (belatacept; CTLA-4-Ig) only (9).

This book also includes a short collection of articles on cellbased therapies in SSc (Maria et al.; Peltzer et al.; Maria et al.; Rozier et al.), and we would like to shortly discuss the progress of knowledge in this field, more precisely on MSC-based therapies. The questions concerning the source and origin of MSC are still under debate, in particular the use of an autologous versus allogeneic approach (23). Concerning MSC and SSc, 12 studies are registered today on the site "ClinicalTrials.gov", with several distinct methodological approaches. So, we can expect to get answers to many questions in the months and years to come. Another perspective in the field of MSC is represented

\section{REFERENCES}

1. Allanore Y, Simms R, Distler O, Trojanowska M, Pope J, Denton CP, et al. Systemic Sclerosis. Nat Rev Dis Primers (2015) 1:15002. doi: 10.1038/ nrdp. 2015.2

2. Elhai M, Avouac J, Kahan A, Allanore Y. Systemic Sclerosis: Recent Insights. Joint Bone Spine (2015) 82:148-53. doi: 10.1016/j.jbspin.2014.10.010

3. Laurent P, Allard B, Manicki P, Jolivel V, Levionnois E, Jeljeli M, et al. Tgf $\beta$ Promotes Low IL10-Producing ILC2 With Profibrotic Ability Involved in Skin Fibrosis in Systemic Sclerosis. Ann Rheum Dis (2021). doi: 10.1136/ annrheumdis-2020-219748

4. Ponsoye M, Frantz C, Ruzehaji N, Nicco C, Elhai M, Ruiz B, et al. Treatment With Abatacept Prevents Experimental Dermal Fibrosis and Induces Regression of Established Inflammation-Driven Fibrosis. Ann Rheum Dis (2016) 75:2142-9. doi: 10.1136/annrheumdis-2015-208213

5. Boleto G, Guignabert C, Pezet S, Cauvet A, Sadoine J, Tu L, et al. T-Cell Costimulation Blockade Is Effective in Experimental Digestive and Lung Tissue Fibrosis. Arthritis Res Ther (2018) 20:197. doi: 10.1186/s13075-018-1694-9

6. Elhai M, Meunier M, Matucci-Cerinic M, Maurer B, Riemekasten G, Leturcq $\mathrm{T}$, et al. Outcomes of Patients With Systemic Sclerosis-Associated by their extracellular vesicles (EVs), that function as mediators of intercellular communication and natural transporters of bioactive molecules (proteins, RNA, DNA) to their microenvironment or systemically. Actually, MSC-derived EVs exhibit the main functions of MSC, and thus could constitute an attractive alternative to MSC, i.e. not suffering from the potential issues associated with expanded cells. In addition, EVs possess the advantage to be safer (anuclear) and could be stored at room temperature as lyophilized product (10). Recently, our group demonstrated the beneficial effects of EVs obtained from adipose tissue-derived MSC (ASC), both in vivo in the $\mathrm{HOCl}$-induced SSc model (11) and in vitro in a TGF $\beta 1$-induced model of human myofibroblasts, mimicking the characteristics of fibroblasts isolated from SSc patients (12). We also demonstrated that miR-29a-3p (contained in the EVs) plays a pivotal role for the therapeutic effects of ASC, probably by targeting fibrotic, remodeling, apoptotic, and epigenetic processes (11). These findings make even more sense when considering the different key players and mechanisms described in this book. Finally, in parallel with the development of pharmacological molecules, research on cell therapies is also progressing, with interesting therapeutic prospects using the cells or their derived vesicles.

SSc is definitely a complex disease with many key players, which for a long time escaped our understanding and, which we know better, day by day. The challenge of SSc remains still very important, both pathophysiologically and therapeutically. We hope you enjoy reading this book. We hope that it will open up new perspectives for all SSc specialists, and also for all others in their own fields of research.

\section{AUTHOR CONTRIBUTIONS}

All three authors contributed to the editing of the Research Topic, writing and reviewing of the present Editorial article. All authors contributed to the article and approved the submitted version.
Polyarthritis and Myopathy Treated With Tocilizumab or Abatacept: A EUSTAR Observational Study. Ann Rheum Dis (2013) 72:1217-20. doi: 10.1136/annrheumdis-2012-202657

7. de Paoli FV, Nielsen BD, Rasmussen F, Deleuran B, Søndergaard K. Abatacept Induces Clinical Improvement in Patients With Severe Systemic Sclerosis. Scand J Rheumatol (2014) 43:342-5. doi: 10.3109/03009742.2013. 812238

8. Khanna D, Spino C, Johnson S, Chung L, Whitfield ML, Denton CP, et al. Abatacept in Early Diffuse Cutaneous Systemic Sclerosis: Results of a Phase II Investigator-Initiated, Multicenter, Double-Blind, Randomized, PlaceboControlled Trial. Arthritis Rheumatol (2020) 72:125-36. doi: 10.1002/ art.41055

9. Adom D, Dillon SR, Yang J, Liu H, Ramadan A, Kushekhar K, et al. ICOSL+ Plasmacytoid Dendritic Cells as Inducer of Graft-Versus-Host Disease, Responsive to a Dual ICOS/CD28 Antagonist. Sci Transl Med (2020) 12: eaay4799. doi: 10.1126/scitranslmed.aay4799

10. Maumus M, Rozier P, Boulestreau J, Jorgensen C, Noël D. Mesenchymal Stem Cell-Derived Extracellular Vesicles: Opportunities and Challenges for Clinical Translation. Front Bioeng Biotechnol (2020) 8:997. doi: 10.3389/ fbioe.2020.00997 
11. Rozier P, Maumus M, Maria ATJ, Toupet K, Lai-Kee-Him J, Jorgensen C, et al. Mesenchymal Stromal Cells-Derived Extracellular Vesicles Alleviate Systemic Sclerosis Via miR-29a-3p. J Autoimmun (2021) 121:102660. doi: 10.1016/j.jaut.2021.102660

12. Rozier P, Maumus M, Bony C, Maria ATJ, Sabatier F, Jorgensen C, et al. Extracellular Vesicles Are More Potent Than Adipose Mesenchymal Stromal Cells to Exert an Anti-Fibrotic Effect in an In Vitro Model of Systemic Sclerosis. Int J Mol Sci (2021) 22:6837. doi: 10.3390/ijms22136837

Conflict of Interest: The authors declare that the research was conducted in the absence of any commercial or financial relationships that could be construed as a potential conflict of interest.
Publisher's Note: All claims expressed in this article are solely those of the authors and do not necessarily represent those of their affiliated organizations, or those of the publisher, the editors and the reviewers. Any product that may be evaluated in this article, or claim that may be made by its manufacturer, is not guaranteed or endorsed by the publisher.

Copyright (c) 2021 Guilpain, Noël and Avouac. This is an open-access article distributed under the terms of the Creative Commons Attribution License (CC BY). The use, distribution or reproduction in other forums is permitted, provided the original author(s) and the copyright owner(s) are credited and that the original publication in this journal is cited, in accordance with accepted academic practice. No use, distribution or reproduction is permitted which does not comply with these terms. 